\title{
PHASE TRANSITIONS AND METASTABLE STATES IN THE PRESENCE OF RANDOM FIELDS
}

\author{
R. A. Cowley \\ Department of Physics BNL- -38690 \\ University of Edinburgh, \\ Mayfield Road \\ DE87 001471 \\ Edinburgh, Scotland \\ R. J. Birgeneau \\ Department of Physics \\ Massachusetts Institute of Technolczy \\ Cambridge, MA 02139 \\ G. Shirane \\ Brookhaven National Laboratory, \\ Department of Physics \\ Upton, N.Y. 11973.
}

The application of a unfform magnetic field to a diluted Ising

antiferromagnet produces a random staggered field and so can be used to study the randon field Ising model. In this paper we describe neutron scattering measurements on $\mathrm{Mn}_{x} \mathrm{Zn}_{1-x} \mathrm{~F}_{2}$. They show that at

low temperatures there are many metastable states which are all stable

for experimentally accessible times. There is a sharp boundary in the

H/T plane wich separates metastability from ergodicity. The critical

phenomena close to this metastability boundary is discussed.

\section{DISCLAIMER}

This report was prepared as an account of work sponsored by an agency of the United States Government. Neither the United States Government nor any agency thereof, nor any of their employees, makes any warranty, express o: implied, or assumes any legal liability or responsibility for the accuracy, completeness, or usefuliness of any information, apparatus, product, or process disclosed, or represents that its use would not infringe privately owned rights. Reference herejn to any specific commercial product, process, or service by trade name, trademark, manufacturer, or otherwise does not necessarily constitute or imply its endorsement, recommendation, or favoring by the United States Government or any agency thereof. The views and opinions of authors expressed herein do not necessarily state or reflect those of the United States Government or any agency thereof.

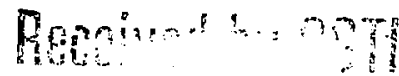


The randow field Ising model (RFIM) is one of the simplest problems with competing interactions. The exchange interactions act so as to produce long range order, but the ragnetic fields, which are randomly directed at each site, act so as to destroy the order. Ever since the work of Inry and $\mathrm{Ma}^{1}$, it has been realized that random fields can produce dramatic effects on phase transitions and ordering. The RFIM has proved, however, to be a more complicated problem than was initially anticipated by efther the theorists or the experimentalists, and despite a large body of work, it is still not fully understood.

A very conventent experimental realization of the random field Ising model is obtained by the application of a unfform field to a diluted antiferromagnet ${ }^{2}$; here the uniform field produces a random staggered field due to the randomess in the Zeeman energy. Over the paģt 6 years a large number of experiments employing a variety of experimental techniques has been performed using $d=2$ and $d=3$ antiferromagnets. The progress up until 1983 was reviewed at the preceding Statistical Physics Conference ${ }^{3}$. In this brief review we discuss the progress made since then with emphasis on results obtained using neutron scattering techniques with the $d=3$ syetem $M n_{x} \mathrm{Zn}_{1-x_{2}} \mathrm{~F}^{4}$. Very similar results have also been observed with $F e_{x} \mathrm{Zn}_{1-x_{2}}{ }^{5}$

In all of the neutron scattering experiments on the diluted $d=3$ systems, it is found that when the samples are cooled in a field, FC, long range order is not established at low temperature, but the 
scattering profiles have a characteristic Lorentzlan squared form as a function of wavevector. In contrast, if the samples are cooled in the absence of the field, and the fleld applied, ZFC, long range order is retained until the sample is heated above a well defined temperature. Figure 1 1llustrates this difference at a temperature just $0.4 \mathrm{~K}$ below this metastability boundary, $T_{M}(H)$. Much of the recent work has been performed with a view to elucidating the behavior close to this metastability line. Differences in the FC and ZFC behavior are also observed in macroscopic measurements such as thermal expansion and magnetization. 6

It is now generally belleved that in equilibrium the lower critical dimension of the random field Ising model is 27 . Consequently systems with $d=3$ are predicted to have a long range ordered ground state, and there have been many theories ${ }^{8}$ of the critical phenomena describing the possibly continuous phase transition into this long range ordered phase at $\mathrm{T}_{\mathrm{N}}(\mathrm{H})$. Because none of these theorles explain the observed metastabillty, models have also been developed ${ }^{9}$ to explain this metastability as arlsing from anomalously large barrlers to the motion of domain walls between the antiferromagnetic domain... These theortes predict that the size of the domains should increase with time as $\ln (t / \tau)$ where $\tau$ is a microscopic time. It is important to emphasize that $\mathrm{Mn}_{\mathrm{x}} \mathrm{Zn}_{1-\mathrm{x}^{F}}$ has only a very small spln wave energy gap: $-1 \mathrm{~K}$ at $7 \mathrm{~T}$ and $40.0 \mathrm{~K}$. The metastabilfty cannot, therefore, arise from a thermil freezing of the system as there are very many spin waves excited at the relevant temperatures. To probe the predicted time dependence of the domain 
size the scattering was measured as a function of time after cooling as rapldiy as possible from above to below the metastability boundary. Typical results are shown in Fig. 1. It was found that the size of the domalns at $900 \mathrm{mins}$ was $1.01 \pm 0.03$ times that at 8 mins, whereas with any reasonable microscopic time, $\tau$, the logarithm would. predict a minimum ratio of 1.14. Similar experiments have been performed by quenching the ffeld as well as the temperature in $\mathrm{Mn}_{0.5} \mathrm{Zn}_{0.5} \mathrm{~F}_{2}$ with similar results ${ }^{4}$. We conclude that the FC state does not decay logarithmically with time.

In an attempt to understand the observed FC behavior in the critical region $V 111 a^{10}$ and $F$ isher ${ }^{10}$ have suggested that the barrier heights for domain wall motion diverge just above $T_{N}(H)$. Further, the barrier height varies sufficiently rapidly with $T-T_{N}(H)$, in these theories, that one expects a quite well-defined metastability boundary. ' Close to this metastabjlity boundary $\mathrm{KIng}$ et al. 11 , have 1ndeed found that the a. c. susceptibility is frequency dependent at. anomalously 10w frequencles. We conclude that anomalously slow dynamics can account for the metastability boundary.

Desplte this result, the properties of the metastable phase are not get fuliy understood. Fig. 2 shows that the FC Inverse correlation length continues to decrease on cooling below the boundary; thus the system is not completely frozen. Secondly, if the fleld is reduced in the metastable region the system rapidly relaxes to the same state as that achieved by the FC procedure in the new field. In contrast, when the field is raised no contraction of the domains occurs. This asymmetry in the behaviour is possibly consistent with the theories, but it is surprising that the relaxation 
Is Independent of temperature, and that the same FC state is always reached from different more disordered configurations.

If the metastability transition at $T_{M}(H)$ occurs above a continuous phase transition at $T_{N}(H)$ to long range order, the behaviour of the scattering above the metastability may give information about the exponents to be expected at the continuous phase transition. Fig. 3 shows the inverse correlation length for various flelds as a function of temperature. For $H=0$ the correlation length exponent is $v=0.71 \mathrm{in}$ agreement with theory for the random exchange Ising model. However fits to the data for higher flelds give exponents between 1.0 and 1.9 depending on the fleld and range over which the data are fitted. The results obtained ${ }^{5}$ in $F e_{x^{2}} n_{1-x_{2}} F_{2}$ are very simflar to those of $M n_{x} Z n_{1-x} F_{2}$. It is therefore very difficult if not impossible to extract these exponents reliably, without a theoretical prediction for the relevant crossover functions. The amplitude of the Lorentzian squared, shown In F1g. 4, Increases steadily as the sample is cooled to the metastability temperature. This behavior also occurs at other fields. If there is a continuous phase transition, this Lorentzian squared term must evolve continuousiy into the Bragg reflection from the long range order. If $B>0$, this requires that the amplitude vary continuousi; to zero at the phase transition temperature (that is $\bar{\pi}>1$ ) In disagreement with the trend evident in Fig. 4. 
Alternatively the amplitude could tend to a constant at $T_{N}$ in which case $\beta=0$. On the other hand a conventional critical point analysis of the data above the metastability temperature suggests that the amplitude diverges at $T_{N}(H),(\bar{n}<1)$ which is inconsistent if the transition is continuous. We conclude that our data suggest that if there is a transition to long range order it is probably of first order ${ }^{12}$, even if very weakly so.

We have studied the behavior of the ZFC state close to the metastability boundary. With increasing temperature just below the boundary there is a Lorentzian squared component to the scattering whose Intensity increases, whlle the inverse correlation length decreases until the metastability boundary is reached. At that point they have the same values as obtained in the FC experiments. The long range order decreases to zero over a narrow, $-0.4 \mathrm{~K}$, temperature interval at the metastability boundary. We conclude that ZFC state undergoes an abrupt, possibly first order transition at the metastability boundary to a state identical with that obtalned by the FC procedure. It should be emphasized that in $\mathrm{H}=0$ in our $M n_{0.75} Z_{0.25} F_{2}$ sample the rounding of $T_{N}$ due to Inhomogenet1es is less than $0.025 \mathrm{~K}$.

In conclusion, the random field Ising model has been found to have many unexpected features. At low temperatures there are many metastable states and the theory of these states is not yet completely convincing. It is predicted that if the system remained in equilibrium and the metastability did not occur, there would be a 
transition to a state with long range order. Our result's suggest that this transition, if tt occurred, would be of first order. In the future we can expect to see further 1mprovements and more detalled experiments over many decades of frequency close to the metastability boundary. These experiments w11 hopefully help elucidate not only the metastability found in the random field Istng model, but also similar effects found in other systems with competing interactions. ACKNOWLEDGEMENTS

R.A.C. Is grateful for the hospitality of Brookhaven National Laboratory where much of this work was done. We are grateful to our collaborators, Prof. H. Yosh1zawa and Dr, Y. Y. Uemura.

Work at Brookhaven supported by the Division of Materials Sciences, U.S. Department of Energy under contract DE-AC02-76CH00016 and at MIT by the U.S. National Science Foundation - Low Temperature Physlcs program, contract No. DMR 85-01856 and at Edinburgh by the SERC. 


\section{References}

1. Y. Imry and S. K. Ma, Phys. Rev. Lett. 35 (1975) 1399.

2. S. Fishman and A. Aharony, J. Phys. C 12 (1979) L729.

3. R. J. Birgeneau, R. A. Cowley, G. Shirane and H. Yoshizawa, J. Stat. Phys. $34,(1984) 817$.

4. R. J. Birgeneau, R. A. Cowley, G. Shirane, and H. Yoshlzawa, Phys. Rev. Lett. 54 (1985) 2147.

R. J. Birgeneau, Y. Shapira, G. Shirane, R. A. Cowley, and H. Yoshizawa, Phys1ca 137B (1986) 83 and R. A. Cowley, G. Shirane, H. Yoshizawa, Y. J. Uemura, and R. J. Birgeneau (to be published).

5. R. A. Cowley, H. Yosh1zawa, G. Sh1rane, and R. J. Birgeneau, Z, für Phystk 58 (1984) 15.

D. P. Belanger, A. R. KIng, and V. Jaccarino, Phys. Rev. B31 (1985) 4538.

H. Yoshizawa, R. A. Cowley, G. Shirane and R. J. Birgeneau, Phys. Rev. B31 (1985) 4548 .

6. Y. Shapira, N. F. O1iveira and S. Foner, Phys. Rev. B30, 6639 (1984).

7. J. Z. Imbrie, Phys. Rev. Lett. 53 (1984) 1747 and this volume.

8. For example:

A. Aharony, Y. Imry, and S. R. Ma, Phys. Rev. Lett. 37 (1976) 1364.

G. Paris1 and N. Sourlas, Phys. Rev. Lett. 43 (1978) 744. 
M. Schwartz, J. Phys. C18 (1985) 135.

Y. Shap1r, Phys. Rev. Lett. 54 (1985) 154.

A. T. Bray and M. A. Moore, J. Phys. C 18 (1985) L927.

A. N. Berker and S. R. McKay, Phys. Rev. B33 (1986) 4712.

9. J. Villain, Phys. Rev. Lett. 54 (1984) 1543

G. Grinsteln and J. Fernandez, Phys. Rev. B29 (1984) 6389.

10. J. Villain, J. de Physique 46 (1985) 1843.

D. S. Fisher, Phys. Rev. Lett. 56 (1986) 416.

11. A. R. King, J. A. Mydosh and V. Jaccar1no, Phys. Rev. Lett. 56 (1986) 2525.

12. A. P. Young and M. Nauenberg, Phys. Rev. Lett. 54 (1985) 429. 


\section{Figure Captions}

F1g. 1 The wavevector dependence ${ }^{4}$ of the neutron scattering from $\mathrm{Mn}_{0.75} \mathrm{Zn}_{0.25} \mathrm{~F}_{2}$. The fits are to a Lorentzian squared profile FC or to a Lorentzian squared and a Gaussian to describe the Bragg reflection, ZFC.

Fig. 2 The inverse correlation length obtained from neutron

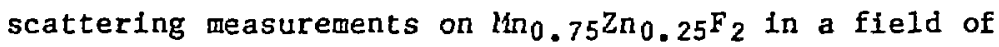
6.5T. The dotted Iine is the metastability boundary.

Fig. 3 The inverse correlation length above the metastability boundary for varlous flelds in $\mathrm{Mn}_{0.75} \mathrm{Zn}_{0.25} \mathrm{~F}_{2}$.

Fig. 4 The amplitude of the Lorentzian squared derived from neutron scattering tpeasurements on $\mathrm{Mn}_{0.75} \mathrm{Zn}_{0.25} \mathrm{~F}_{2}$ in $6.5 \mathrm{~T}$. The dotted line gives the metastabillty boundary. 


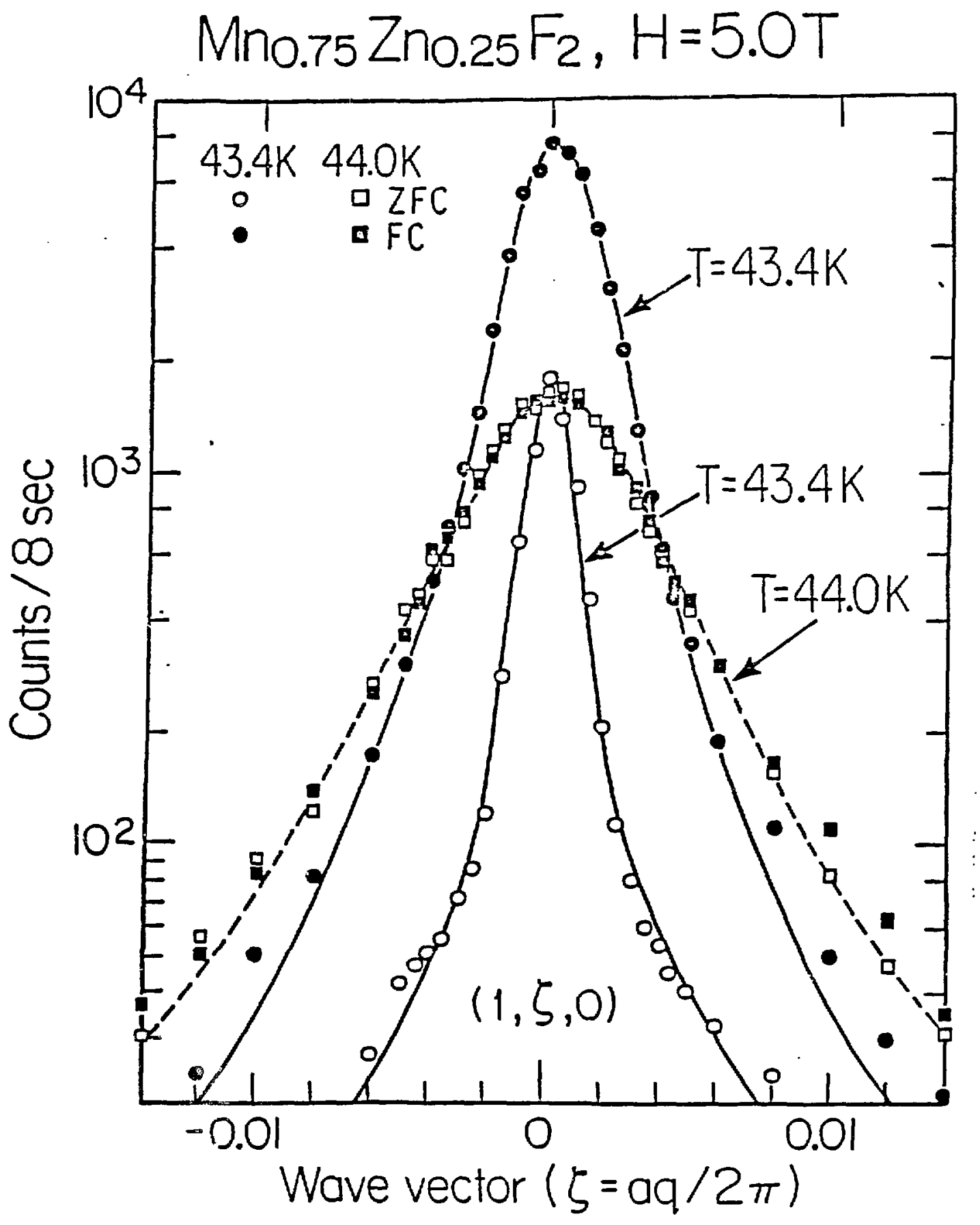

Figure 1 


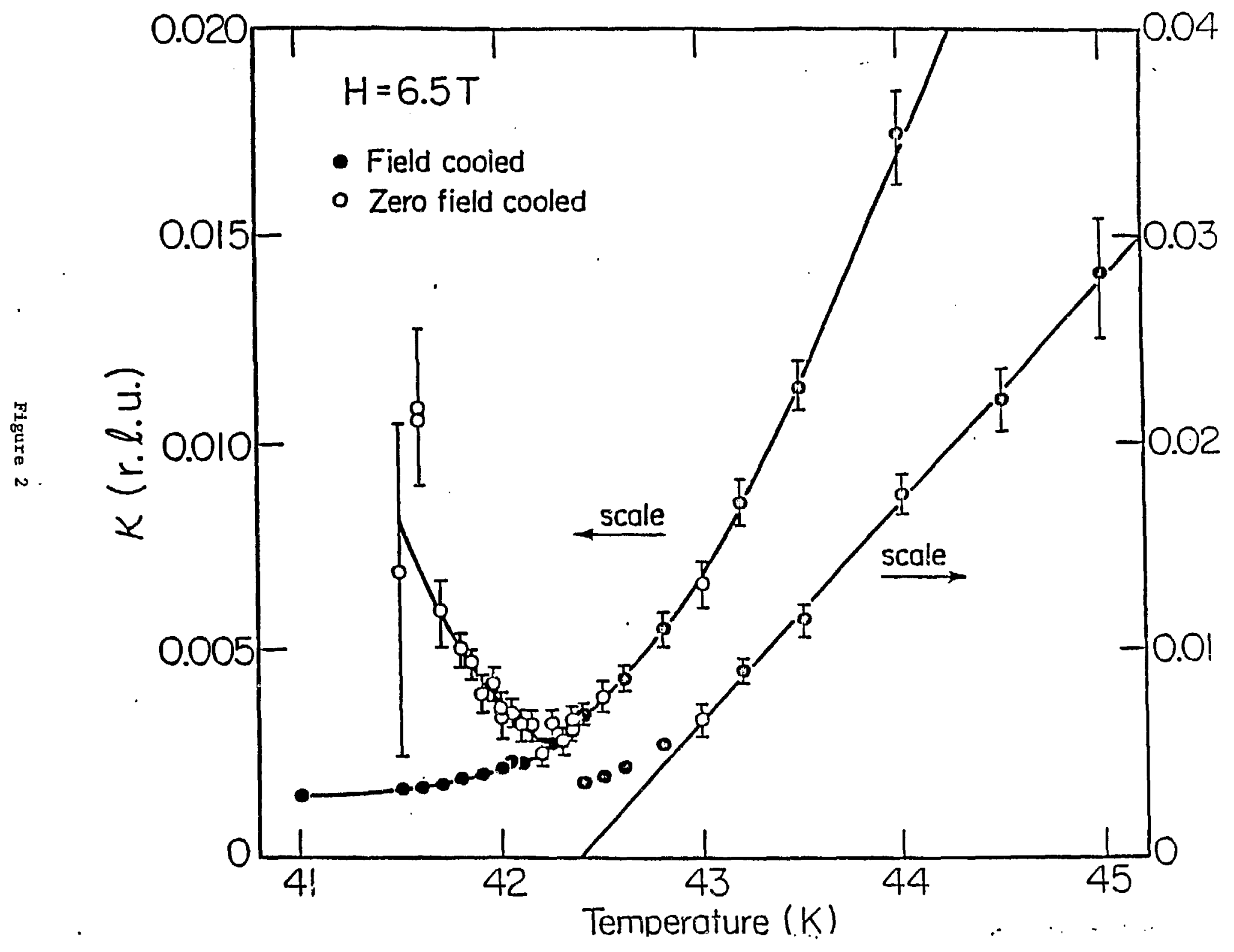




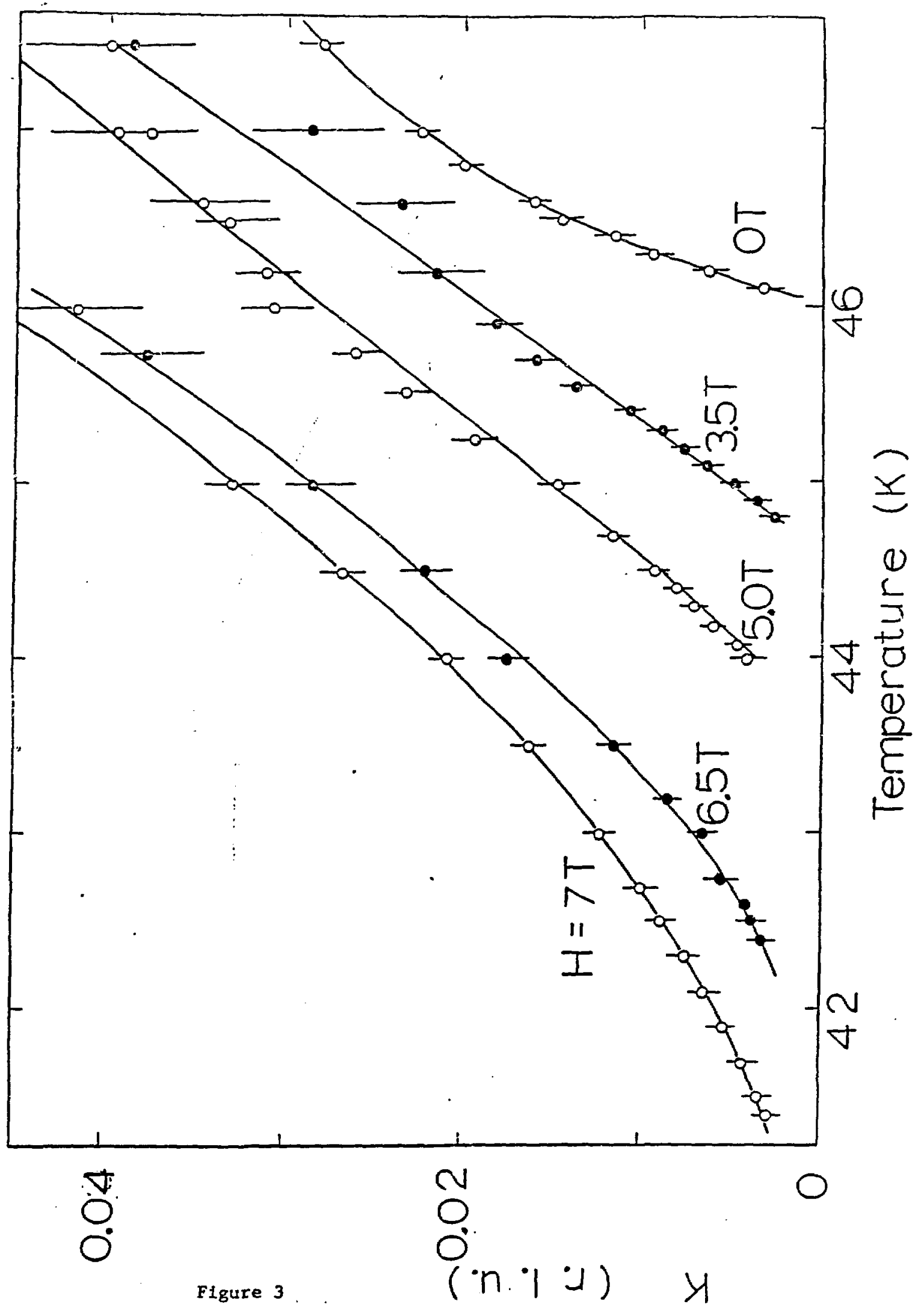




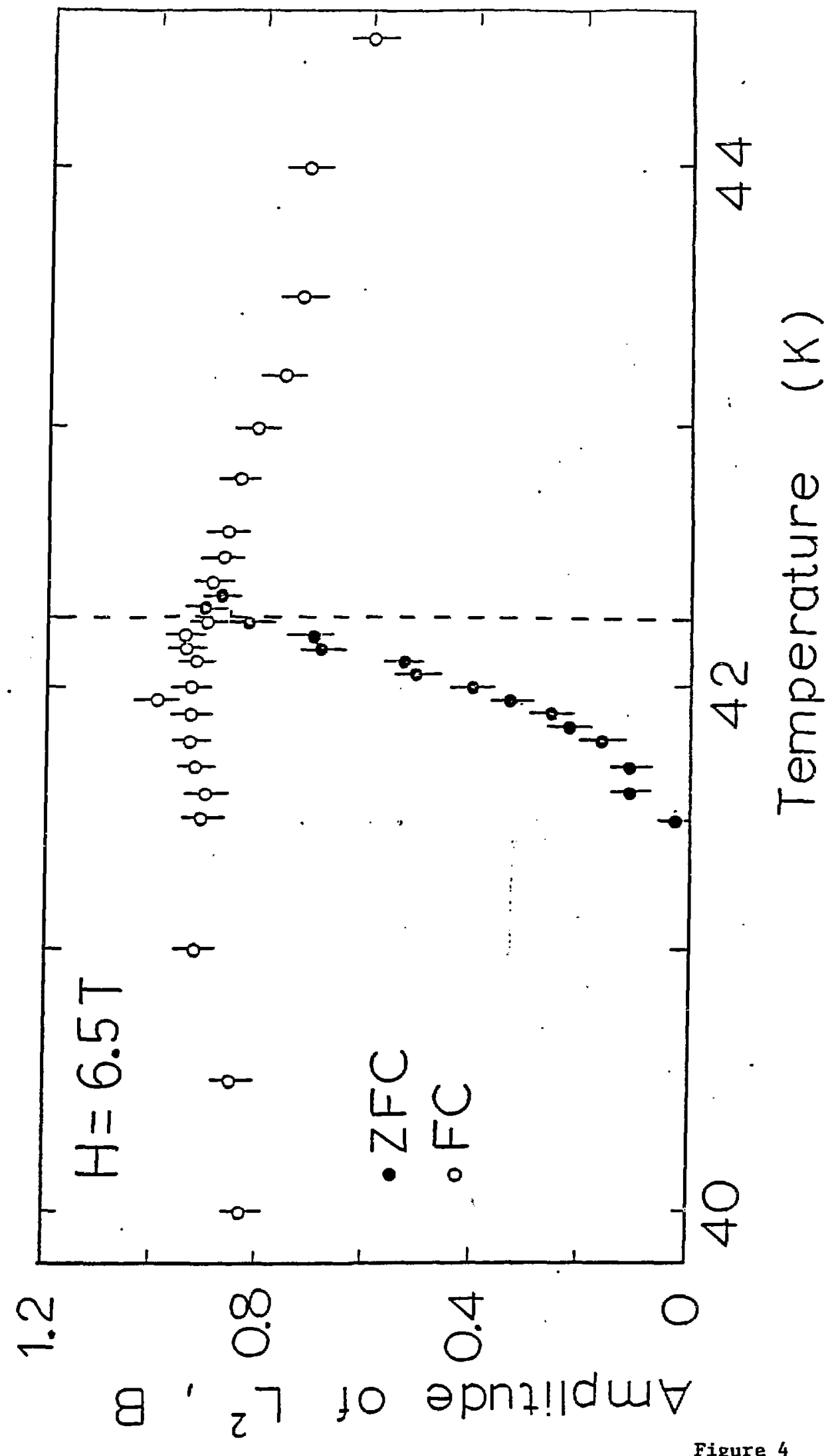

Figure 4 